\title{
IRON DEPOSITION IN THE BRAIN FOLLOWING THE ISCHEMIA IN A RAT MODEL OF ISCHEMIC TOLERANCE
}

\begin{abstract}
Viera Danielisová, Miroslava Némethová, Jozef Burda
Slovak Academy of Sciences, Institute of Neurobiology, Košice, Slovak Republic: Department of Neurochemistry

Summary: Preconditioning of the brain by short-term ischemia increases brain tolerance to the subsequent severer ischemia. In this study, we investigated iron deposition in the cerebral cortex and the ischemic tolerance in a rat model of cerebral ischemia. Forebrain ischemia was induced by four-vessel occlusion for $5 \mathrm{~min}$ as ischemic preconditioning. Two days after preconditioning or after the sham-operation, the second ischemia was induced for $20 \mathrm{~min}$. Changes in the cerebral cortex were examined after 1 to 8 weeks of recirculation following $20 \mathrm{~min}$ ischemia with or without preconditioning using the iron histochemistry. Granular deposits of the iron were found in the cytoplasm of the pyramidal cells in the layers $\mathrm{III}$ and $\mathrm{V}$ of the frontal cortex after 1 week of recirculation. When the rats were exposed to $5 \mathrm{~min}$ ischemia 2 days before 20 min lasting ischemia, the deposition of iron in the cytoplasm of the pyramidal cells in layers III and $\mathrm{V}$ of the frontal cortex was significantly lower during all periods of reperfusion. Preconditioning $5 \mathrm{~min}$ ischemia followed by 2 days of reperfusion before $20 \mathrm{~min}$ ischemia also prevented degeneration of the pyramidal neurons in layers III and $\mathrm{V}$ of the frontal cortex.
\end{abstract}

Key words: Cerebral ischemia; Ischemic tolerance; Iron; Frontal cortex

\section{Introduction}

Short periods of preconditioning by the ischemia can confer protection against injury which results from longer periods of focal or global ischemia in the central nervous system. Since preconditioning harnesses robust endogenous protective potential of the tissue, elucidation of the mechanisms responsible for the induction of ischemic tolerance may have practical value in the search for effective means of therapeutic intervention in stroke.

Iron is necessary for normal neural function but it must be stringently regulated to avoid iron-induced oxidative injury (1). The recirculation of blood after transient ischemia causes an increase in low molecular weight species of iron, and free radicals formation initiated by iron leads to the brain damage by lipid peroxidation (11). Iron-induced hydroxyl radicals, lipid peroxidation and apoptotic cell death can be blocked by both, endogenously generated and exogenously administered nitric oxide. The ischemic preconditioning induces expression of stress proteins such as hemeoxygenase- 1 , neuronal nitric oxide synthase, redox factor- 1 and inhibits $\mathrm{p} 66^{\text {shc }}$, as well as hypothermia therapy suppresses the generation of toxic reactive oxygen, lipids and thiol species evoked by bioactive iron complexes in the brain (4).

Free iron, more than any other transition metal, has been implicated in redox transitions and consequential ge- neration of oxygen free radicals. The iron regulatory proteins, which control other Fe-binding proteins and thus the regulation of cellular iron metabolism also play a role in decreasing the reactive oxygen species generating capacity of $\mathrm{Fe}(2)$.

There were many studies dealing with the role of iron in neuronal damage during early stages of cerebral ischemia (6), but abnormal iron deposition at a late stage after transient cerebral ischemia has not been reported except in the histochemical studies of the spatial and temporal distributions of the iron (5), ferritin, and transferrin (as iron binding proteins), and of the astroglial and microglial reactions at $1-24$ weeks after transient forebrain ischemia (8).

In the present study, we investigated postischemic neuronal damage in the layer $\mathrm{V}$ of the cerebral cortex. We examined abnormal iron deposition in the brain during the chronic phase after ischemia, using the intensification of Perl's histochemical reaction for ferric iron by 3,3'-diamonobenzidine (DAB). We also assessed the effectiveness of ischemic tolerance induced by preconditioning with respect to the cell damage and iron deposition.

\section{Materials and Methods}

The experiments were done in agreement with the Slovak Law of Animal Protection No. 115/1995 and under the supervision of the Institutional Ethical Committee for ani- 
mal research. 8- to 10-weeks-old male rats of the Wistar strain were used. The rats' weight was 250-300 g at the beginning of the experiment. They were placed on a deprivation schedule to maintain their weights at approximately $80 \%$ of the free-feeding level. The rats were housed in groups of four per cage under the constant temperature $\left(23 \pm 2^{\circ} \mathrm{C}\right)$ and a 12-h light/dark cycle (light period: 07.00-19.00 h), with water freely available. Transient forebrain ischemia was induced by the four vessel occlusion method (4-VO) described by Pulsinelli and Brierley (9) with modifications (10). Briefly, the rats were anaesthetised i.p. by ketamine $(100 \mathrm{mg} / \mathrm{kg}$ body wt) and xylozine (15 $\mathrm{mg} / \mathrm{kg}$ body $\mathrm{wt}$ ). Vertebral arteries were electrocauterized through the alar foramen of the first cervical vertebra. Both common carotid arteries were exposed through a ventral midline cervical incision and ligatures were placed loosely around each artery without interrupting the carotid blood flow. Next day, under light fluothane anaesthesia both common carotid arteries were reexposed and occluded with aneurysmal clips to induce forebrain ischemia. The arteries were occluded for $5 \mathrm{~min}$ (ischemic preconditioning) and 2 days later the carotid arteries were occluded for $20 \mathrm{~min}$ again. At the end of 20 min lasting bilateral carotid occlusion, blood flow was restored by releasing the clips. The rats which became unresponsive and lost the righting reflex during bilateral carotid artery occlusion, and did not reveal seizures during and after the ischemia were retained in the experiment. Only such animals can be considered to fulfil the criteria of adequate ischemia. Criteria of forebrain ischemia were the following: the bilateral loss of the righting reflex, paw extension, and the mydriasis. Sham-operated animals ( $\mathrm{n}=4$ in each group) were treated in a same manner but without the occlusion of the common carotid arteries. Rectal temperature of animals was maintained at $37^{\circ} \mathrm{C}$ during the surgery and following ischemia by the heating pad and lamp. The animals were sacrificed at 1, 4 and 8 weeks after the preconditioning ischemia or after the second ischemia.

At 1,4 and 8 weeks of recirculation, the animals $(n=6$ in each group) were deeply anaesthetised i.p. by the above mentioned mixture of ketamine and xylozine and then perfused transcardially with saline, followed by $4 \%$ parafor-
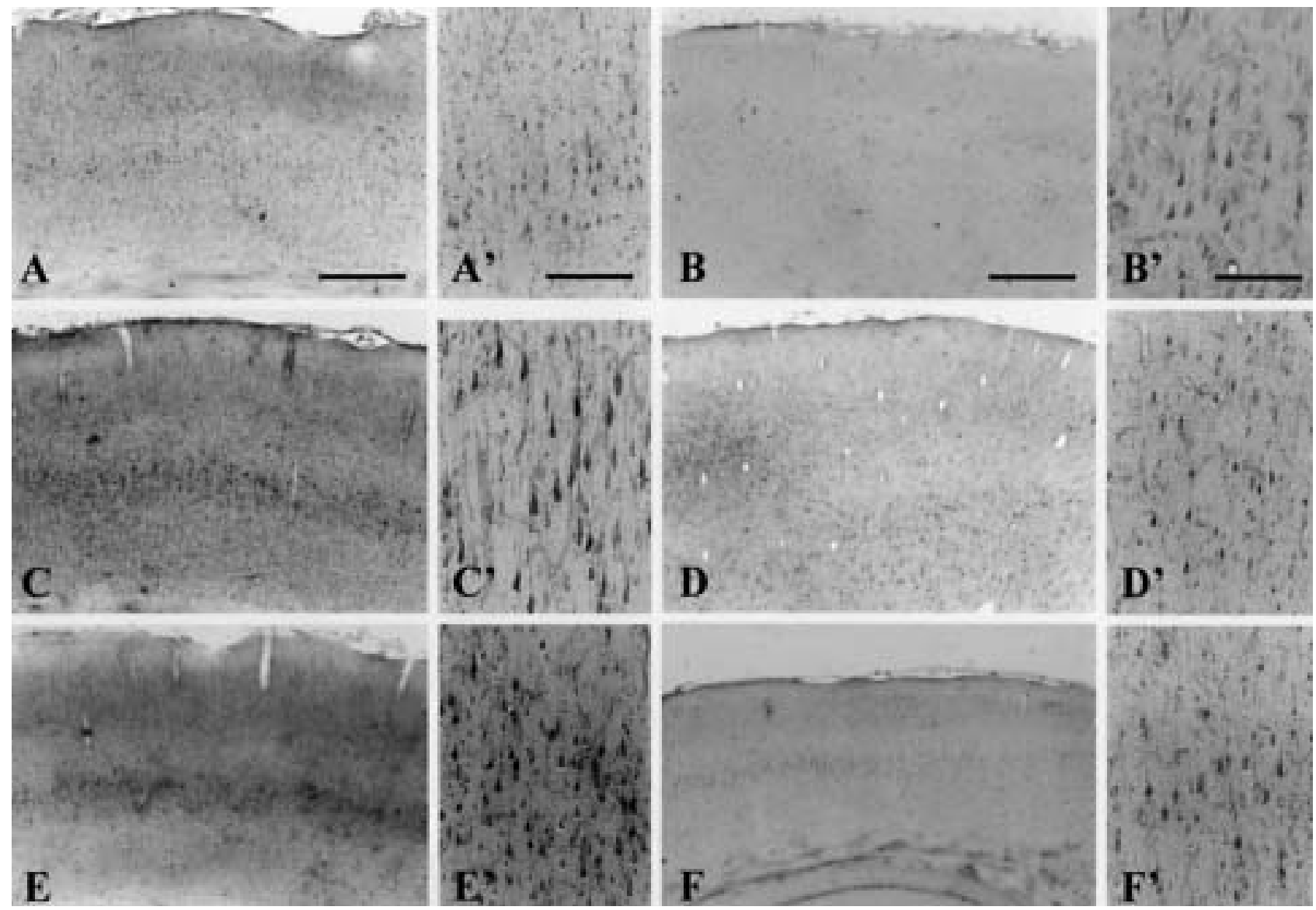

Fig. 1: Photomicrographs of Perl's reaction at a level of frontal cortex without preconditioning after 1 week (A and A'), 4 weeks (C and C'), 8 weeks (E and E') of reperfusion, and with preconditioning after 1 week (B and B'), 4 weeks (D and D'), 8 weeks (F and F') of reperfusion. Scale bar: $400 \mu \mathrm{m}$ (A, B, C, D, E, F) and 40 um (A', B', C', D', E', F'). 
maldehyde solution with $0.1 \mathrm{M}$ phosphate buffer (PB; pH 7.4). The brains were removed from the skull, and postfixed for $3 \mathrm{~h}$ in the same fixative. The brains were cut coronally on a vibratome to $30 \mu \mathrm{m}$. The sections were stained with hematoxylin-eosin and by Perl's reaction with or without 3,3'-diaminobenzidine (DAB) intensification (7). Sections were well washed with deionized water for at least $30 \mathrm{~min}$, incubated in Perl's solution ( $1 \%$ potassium ferrocyanide/ $1 \%$ $\mathrm{HCl}$ ) at the room temperature for $30 \mathrm{~min}$ followed by washing with deionized water for $30 \mathrm{~min}$. Sections without DAB intensification were counterstained with neutral red and mounted. For the intensification of Perl's reaction, the sections were incubated for $20 \mathrm{~min}$ in $0.5 \% \mathrm{DAB}$ in $0.1 \mathrm{M} \mathrm{PB}$ and for $15 \mathrm{~min}$ in the same medium with $0.005 \% \mathrm{H}_{2} \mathrm{O}_{2}$. The reaction was finished by washing with deionized water for $30 \mathrm{~min}$. Some sections were counterstained with methyl green. Control slides were amplified with DAB but without the preincubation with Perl's solution. No positive staining was found in any of control slides.

Statistical significance of differences between the groups was analysed using one-way ANOVA test followed by posthoc Duncan's test. The result $\mathrm{p}<0.05$ was considered to be significant.

\section{Results}

Ischemic changes in neurons induced by transient forebrain ischemia were evaluated on sections of the frontal cortex stained with hematoxylin and eosin. In this model, neuronal damage was observed only in the layers III-V of the cerebral cortex. The effect of preconditioning was visible already after 1 week of recirculation.

In sham-operated control rats, the iron was occasionally present in the perikarya and neuronal processes of some

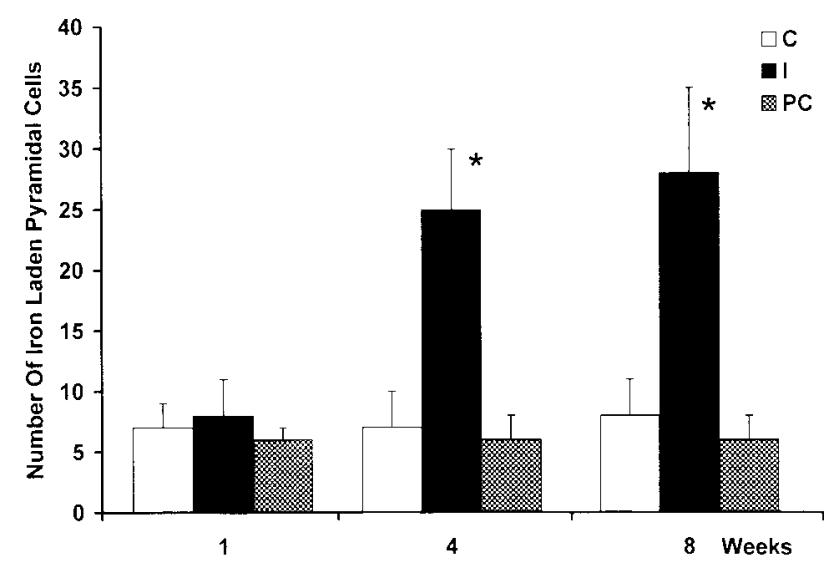

Fig. 2: Number of iron-positive pyramidal neurons in layer $\mathrm{V}$ of frontal cortex. Neurons were counted using a light microscope at a 50-fold original magnification. An area of $3.0 \times 10^{4} \mu \mathrm{m}^{2}(460 \times 650 \mu \mathrm{m})$ was counted. Values are means $\pm \mathrm{SD}$. ${ }^{*} \mathrm{p}<0.05$ compared to ischemic rats without preconditioning. pyramidal neurons in the layer $\mathrm{V}$ and in a part of the layer III of the frontal cortex in the form of fine granular deposits. The number of iron-containing pyramidal cells in the layer $\mathrm{V}$ of the frontal cortex increased in 1 week and reached maximal values after 8 weeks of recirculation (Figs. 1A, A'; C, C'; E, E'). The injured cellular layer was strongly iron stained (Figs.1E, E'). The protective effect of $5 \mathrm{~min}$ ischemia 2 days before 20 min severe ischemia was visible in layer V of the frontal cortex (Figs. 1B, B'; D, D'; F, F').

Granular iron deposits in pyramidal neurons of the frontocortical layer $\mathrm{V}$ showed significant increase at 4 and 8 weeks compared to ischemic rats without preconditioning (Fig. 2)

\section{Discussion}

The iron deposition in cerebral cortex in a rat model of cerebral ischemia and possible increase of ischemic tolerance of the brain was investigated. We examined the iron deposition in the rat brain during the chronic phase following transient forebrain ischemia with and without preconditioning. The iron began to accumulate after the first week of recirculation in the cytoplasm of the pyramidal cells in the layer $\mathrm{V}$ of the frontal cortex,. Conspicuous iron deposition was seen in the mentioned cells after 8 weeks. Preconditioning with $5 \mathrm{~min}$ ischemia 2 days before $20 \mathrm{~min}$ ischemia preserved the pyramidal cell layer $\mathrm{V}$ of the frontal cortex and produced only a minimal number of iron-containing cells during recirculation.

Abnormally high levels of iron deposition as well as oxidative stress have been demonstrated in a number of neurodegenerative disorders including Alzheimer's disease and those characterised by nigral degeneration such as Parkinson's disease, multiple system atrophy, and progressive supranuclear palsy. Microglia cells are the major sites of iron bound in ferritin and are thought to be partly responsible for oxidative damage in neurodegenerative disorders. Microglia stimulated in vivo with phorbol ester shows increased lipid peroxidation resulting from a superoxide-dependent release of iron from ferritin (12). The interaction of oxidative stress with control of iron metabolism in the brain is confirmed by finding of abnormal iron deposition associated with lipid peroxidation in a transgenic mouse model (with the expression of interleukin IL-6 in astrocytes) of the blood-brain barrier defect associated with progressive neurodegeneration (3).

Preconditioning induction of genes and proteins, capable to prevent or repair the oxidative damage caused by reactive oxygen species, peroxyl lipid radicals and reactive peptidyl thiol radicals could enhance the recovery and repair of partially damaged neurons in the brain. Neuroprotection against cerebral ischemia can be realised if the brain is preconditioned by previous exposure to a brief period of ischemia. Preconditioning of the rat brain by shortterm cerebral ischemia induces resistance to subsequent severe ischemia. 


\section{Conclusions}

This study indicates that preconditioning of the brain by $5 \mathrm{~min}$ ischemia 2 days before $20 \mathrm{~min}$ ischemia prevented neuronal damage by the reduction or limitation of iron accumulation in the layer $\mathrm{V}$ of the frontal cortex. It has been suggested that short ischemic attacks might provide the preconditioning necessary to protect the brain from later, more severe insults. Our data confirm that this indeed might be the case of postischemic iron metabolism.

\section{Acknowledgement}

This study was supported by the Slovak Grant Agency for Science SK-VEGA 2/3219/23.

\section{References}

1. Benkovic SA, Connor JR. Ferritin, transferrin, and iron in selected regions of the adult and aged rat brain. J Comp Neurol 1993;338:97-113.

2. Campbell A, Smith MA, Sayre LM, et al. Mechanisms by which metals promote events connected to neurodegenerative diseases. Brain Res Bull 2001:55:125-32.

3. Castelnau PA, Garrett RS, Palinski W, Witztum JL, Campbell Il, Powell HC Abnormal iron deposition associated with lipid peroxidation in transgenic mice expressing interleukin-6 in the brain. J Neuropathol Exp Neurol 1998;57:268-82.

4. Chiueh CC. Iron overload, oxidative stress, and axonal dystrophy in brain disorders. Pediat Neurol 2001;25:138-47.

5. Danielisová V, Gottlieb M, Burda J. Iron deposition after transient forebrain ischemia in rat brain. Neurochem Res 2002;27:237-42.
6. Davalos A, Real JMF, Soler SS, Molins A, Planas E, Genis D. Iron-related damage in acute ischemic stroke. Stroke 1994;25:1543-6.

7. Hill JM, Switzer RC. The regional distribution and cellular localisation of iron in the rat brain. Neuroscience 1984;11:595-603.

8. Kondo Y, Ogawa N, Asanuma M, Ota Z, Mori A. Regional differences in late-onset iron deposition, ferritin, transferrin, astrocyte proliferation, and microglial activation after transient forebrain ischemia in rat brain. J Cereb Blood Flow Metab 1995;15:216-26.

9. Pulsinelli WA, Brierley JB. A new model of bilateral hemispheric ischemia in the unanesthetized rat. Stroke 1979;10:267-72.

10. Schmidt-Kastner R, Paschen W, Ophoff BG, Hossmann K-A. A modified fourvessel occlusion model for including incomplete forebrain ischemia in rats. Stroke 1989;20: 938-46.

11. Siesjo BK. Mechanisms of ischemic brain damage. Crit Care Med 1988;16 954-63

12. Yoshida T, Tanaka M, Sotomatsu A, Hirai S, Okamoto K. Activated microglia cause iron-dependent lipid peroxidation in the presence of ferritin. Neuroreport 1998;9:1929-33

Submitted March 2004.

Accepted November 2004.

MVDr. Viera Danielisová, CSc., PhD.,

Department of Neurochemistry, Institute of Neurobiology, Slovak Academy of Sciences, Šoltésovej 6, 04001 Košice,

Slovak Republic. e-mail:danielis@saske.sk 